\title{
30 Jahre operative Tätigkeit des Dermatologen - Rückblick, Umblick und Ausblick
}

G. Sebastian

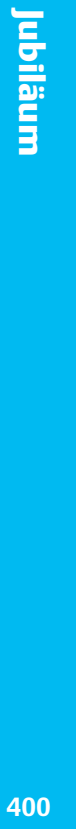

\section{Einleitung}

Ein Beitrag über 30 Jahre operative Dermatologie in Deutschland impliziert automatisch eine Aussage darüber, ob es in diesem Teilbereich unseres Faches Fortschritte gegeben hat, denn jeder Fortschritt wird erst im Rückblick erkennbar. An den Anfang des Beitrages gestellt und herausgehoben werden müssen als entscheidende Meilensteine für die heute in unserem Fach fest verankerte operative und onkologische Dermatologie

- die Gründungen der Vereinigung für Operative Dermatologie (VOD) im Oktober 1977 im „Westen“ und der Sektion Dermatochirurgie (DC) in der Gesellschaft für Dermatologie der DDR im November 1981 „im Osten“,

- die Anerkennung der exakt definierten Inhalte operativer Tätigkeit in der Weiterbildungsordnung für das Fach Haut- und Geschlechtskrankheiten durch die Bundesärztekammer,

- die Aufnahme der Vereinigung für Operative und Onkologische Dermatologie in die Arbeitsgemeinschaft der wissen-

\section{Abstract}

The development of dermatologic surgery during the past 30 years is not restricted to the topics of 25 annual meetings of the VOD. The survey includes biographic aspects of leading persons in the field of operative dermatology especially oncology, phlebology, proctology and laser therapy, as well as aesthetic and corrective aspects. schaftlich-medizinischen Fachgesellschaften (AWMF) im Mai 1994 und

- die kontinuierliche Weiter- und Fortbildung mit 25 Jahrestagungen im deutschsprachigen Raum und 19 Tagungsbänden.

Dem Nachwuchs - der Führung von morgen - soll es Signal und Aufforderung sein, hier weiterzulesen, denn es gibt vieles zu verteidigen und Neues zu erwerben. Der Erhalt des Erreichten und Neuerwerbungen sind längerfristig nur möglich, wenn

- eine Traditionslinie vorhanden ist,

- auf Angriffe von außen auf Bereiche unseres Faches gelassen aber entschieden reagiert wird,

- unser Fach für Innovationen und Alternativen offen ist und

- es gelingt, nicht nur bei den operativ und onkologisch tätigen Dermatologen sondern bei allen Dermatologen auch für diese Bereiche eine „Corporate identity“ erwachsen zu lassen [15].

Institutsangaben

Klinik und Poliklinik für Dermatologie, Universitätsklinikum Carl Gustav Carus an der Technischen Universität Dresden

Korrespondenzadiresse

Prof. Dr. med. Günther Sebastian · Klinik und Poliklinik für Dermatologie · Universitätsklinikum Carl Gustav Carus an der Technischen Universität Dresden · Fetscherstraße 74 - 01307 Dresden ·

E-mail: guenther.sebastian@mailbox.tu-dresden.de

Bibliografie

Akt Dermatol 2004; 30: 400-406 @ Georg Thieme Verlag KG Stuttgart · New York .

DOI 10.1055/s-2004-825879 · ISSN 0340-2541 


\section{Situation der operativen Dermatologie vor 1970}

Die operative und onkologische Dermatologie ist keine Spezialrichtung aus der 2. Hälfte des 20. Jahrhunderts, sondern ein Teil unseres Faches, das sich von den Aufgaben her integrativ und von Anbeginn an gleichberechtigt im breit angelegten Spektrum des neuen Fachgebietes der Haut- und Geschlechtskrankheiten seit der 2. Hälfte des 19. Jahrhunderts entwickelt hat [21]. An dieser Stelle darf angemerkt werden, das operative Dermatologie stets auch onkologische Dermatologie beinhaltet, unbenommen davon, dass sich in den frühen 90er Jahren die Arbeitsgemeinschaft Dermatologische Onkologie (ADO) gründete, um den größten Fortschritt beim malignen Melanom zu initiieren, die multizentrischen, prospektiv-randomisierten Studien [13]. Dass dabei die Wurzeln der Dermatologie sowohl in der Inneren Medizin als auch in der Chirurgie liegen, darüber ist in der Vergangenheit bereits viel geschrieben worden [21]. Zur Herausbildung eines operativen Teils hat nicht allein die „chirurgische Wurzel“ beigetragen sondern die Fülle der Krankheitsbilder, die eine aktive, im weitesten Sinne operative Therapie erforderten. Dazu zählten die chirurgische Versorgung von Lupuskranken, Patienten mit Hauttumoren und „Beinleiden“ bis hin zu den venerologischen Folgekrankheiten. Beredter Ausdruck operativer Tätigkeit des Dermatologen sind die konzipierten und realisierten Baupläne für Operationsräume, z. B. 1892 in Breslau durch A. Neisser, 1896 in Leipzig durch G. Riehl und 1907 in Düsseldorf, um nur einige zu nennen. Mit welcher Weitsicht operative Dermatologie als Schwerpunkt mit dem Ausbau entsprechender operativer Räumlichkeiten in der letzten Dekade des vergangenen Jahrhunderts gefördert wurde, zeigt sich beispielhaft in Tübingen von G. Rassner und in München von O. Braun-Falco und G. Plewig.

Nach dem 2. Weltkrieg entstanden in Ost- und Westdeutschland in den Hautkliniken mit operativer Tradition relativ rasch, mancherorts zögerlich, operative Bereiche, die neben der traditionellen operativen Therapie des Dermatologen ab Mitte der 60er-Jahre verstärkt der operativen Therapie von Hauttumoren den Vorzug gaben. In Dresden durfte der Berichterstatter seit 1967 persönlich und in Kontinuität bis heute den Wandel der operativen und onkologischen Dermatologie einer Hochschul-Hautklinik miterleben und -gestalten. Eindrucksvoll war dabei, wie rasch die im Haus hervorragend betriebene Weichstrahltherapie der Hauttumoren immer mehr zugunsten der Skalpellexzision verlassen wurde. Geprägt wurde diese Entwicklung von Persönlichkeiten, die mit der notwendigen Intention für operative Tätigkeit ausgerüstet waren aber vor allem sehr früh die Bedeutung des operativ-onkologischen Teils der Dermatologie für die zukünftige nationale und internationale Entwicklung des Faches erkannten. Ähnlich, ja gleichartig, verlief dieser Trend in ganz Deutschland. Auf beiden Seiten waren „die Köpfe“ vorhanden [2]. Eine Grenze konnte zwar Dermatologen trennen aber ihr Gedankengut war frei und der Austausch der Ideen konnte nicht blockiert werden. So lief die Bewegung für die Gründung einer operativen Dermatologie als Vereinigung für Operative Dermatologie und später für Operative und Onkologische Dermatologie in Westund für eine Sektion Dermatochirurgie in Ostdeutschland nur wenig zeitversetzt mit dem gleichen Enthusiasmus ab.

\section{Situation der operativen Dermatologie in den 70er-Jahren}

In den frühen 70er-Jahren waren „häufig in der Stille“ in nicht wenigen Hochschul-Hautkliniken und Städtischen Hautkliniken in Ost- und Westdeutschland operative Arbeitsgebiete entstanden. Eine entsprechende Berücksichtigung fand die operative Dermatologie auf nationalen Tagungen jedoch nicht. Deshalb richtete 1975 O. Braun-Falco zusammen mit seinen damaligen Oberärzten G. Burg und B. Konz das „ I. Symposium für Dermatochirurgie“ im Oktober in München aus. H. E. Kleine-Natrop stellte im Juni 1976 das „16. Dresdner Dermatologische Gespräch“ (sein DDG, wie er es hintergründig zu bezeichnen pflegte) unter das Thema „Chirurgisch-operative Therapie von Hautgeschwülsten“. Mit diesen zwei der operativen Tätigkeit des Dermatologen gewidmeten Tagungen waren die Zeichen für die operative Dermatologie auf Öffentlichkeit gestellt. Einen ersten nationalen Auftritt hatte die operative Dermatologie anlässlich der 31. Tagung der DDG im März 1977 in Köln. Hier konnten ihre Vertreter um H. Tritsch einen ganzen Tagungsabschnitt gestalten [18]. Wie bereits ausführlich in München so auch in Köln gaben ihre Vertreter einen Überblick über das in den 70er-Jahren operativ (aktiv) in der Dermatologie Machbare und orientierten sich bei der Auswahl der Themen an der Aktualität und Bedeutung der einzelnen Methoden für die klinische Praxis. Angeboten und demonstriert wurde in München die vorurteilsfreie Zusammenarbeit mit den angrenzenden chirurgisch tätigen Fachgebieten. Zum Ausmaß operativer Tätigkeit wurde als „Grenzfläche“ die Muskelfaszie genannt und ein Operationskatalog, eingebettet in der Weiterbildungsordnung, vorgeschlagen (K. Salfeld 1977). Neben den obligaten Eingriffen wurden wünschenswerte definiert. Für die auf den Gebieten der Phlebologie, Andrologie, Proktologie, Trichologie und korrektiven Dermatologie tätigen Kollegen war es selbstverständlich, ihre Heimat in der operativen Dermatologie zu haben. Die aus der Münchner Tagung entstandene Monographie mit 32 Referaten (B. Konz, G. Burg 1977) darf als Grundlage eines Vergleiches mit der aktuellen Tätigkeit des operativen Dermatologen herangezogen werden und für die Definition von Fortschritten in der operativen Dermatologie gelten.

Auch aus heutiger Sicht ist es verständlich, dass es offensichtlich schwierig war, die prosperierende Entwicklung der operativen Dermatologie unter dem Dach einer entsprechenden Arbeitsgemeinschaft oder Vereinigung darzustellen und wissenschaftlich zu fördern. Für den nicht in die Geburtswehen der Vereinigung für Operative Dermatologie (VOD) involvierten Berichterstatter lesen sich die Aufzeichnungen von damals zu den entscheidenden Schritten fast wie ein Krimi (die von mir hochgeschätzten Initiatoren und damals in den genannten Städten tätigen E. Haneke/Erlangen, M. Hundeiker/Gießen, G. Mahrle/Göttingen, J. Petres/Freiburg, K. Salfeld/Minden, H. Tritsch/Köln und G. Weber/ Nürnberg mögen mir die wenig wissenschaftliche Darstellung verzeihen). Nachdem ein avisierter Termin „geplatzt“ war, fanden sich am 1. Oktober 1977 die genannten Herren - an eine „verschworene Bruderschaft“ erinnernd - im Hause von Herrn G. Weber in Nürnberg zusammen. Diese Gründungsmitglieder beriefen die erste Mitgliederversammlung für Dezember 1977 in den Rosengarten nach Mannheim ein, bei der ein Vorstand und ein wissenschaftlicher Beirat gewählt wurden. Zum Präsidenten der neuen Vereinigung wurde Professor Dr. H. Tritsch gewählt, zum Vizepräsidenten Professor Dr. J. Petres. Schriftführer wurde Professor Dr. M. Hundeiker, Professor Dr. H. Grimmer 
Schatzmeister. Der wissenschaftliche Beirat wurde ebenfalls bestellt und setzte sich aus den Herren Dr. B. Konz, Professor Dr. E. Landes, Professor Dr. Dr. K. Salfeld, Professor Dr. Dr. h. c. U. W. Schnyder und Dr. G. Schwenzer zusammen [25]. Die Satzung, um deren Ausarbeitung sich Hundeiker verdient machte, wurde von den anwesenden 37 Mitgliedern akzeptiert. Dieses Ereignis hat seine Hintergründe, die heute sehr aktuell für unser Gesamtfach sind und an die deshalb mit den Sätzen von H. Tritsch [25] erinnert werden soll: „Trotz oder wegen dieser offensichtlich prosperierenden Entwicklung der operativen Dermatologie und ihres Therapieanteils von ca. 35\% am Krankengut, fehlte es nicht an Versuchen, die Dermatotherapie einseitig auf konservative Methoden zu beschränken, um damit dem Hautarzt den Einsatz der sein Fachgebiet betreffenden chirurgischen Techniken zu verwehren. Eigenartigerweise kamen die Behinderungsversuche nicht nur von außen sondern mitunter auch aus den eigenen Reihen. Hintergrund im Verhalten letzterer Gruppe dürfte vor allem die mangelnde Handfertigkeit, eine unzureichende adäquate Ausbildung und ein dadurch hervorgerufenes Gefühl der Zurücksetzung mit erzwungenen Verzicht im Wettbewerb sein. Treibstoff exogener Widerstände waren, obwohl mit Pseudoargumenten verbrämt, expressis verbis nie artikuliert, letztendlich wirtschaftliche Konkurrenzaspekte anderer Fachbereiche. Versteckt hinter der Behauptung des Unvermögens der Dermatologen zu operativer Tätigkeit, wurde ein alleiniger Kompetenzanspruch mit dem Ziel erhoben, einen wesentlichen Anteil der hautärztlichen Tätigkeit auszugrenzen, um ihn dem eigenen Fachbereich zuordnen zu können. Obgleich die Institutionalisierung der operativen Dermatologie im Hinblick auf diese Gegebenheiten als ein Gebot der Stunde schien, konnten sich weder Berufsverband noch DDG zu entsprechendem Vorgehen entschließen. Zahlreiche Bedenken bestimmten offenbar das Handeln beider Institutionen. Möglicherweise wirkte die Furcht vor streitbaren Auseinandersetzungen mit Vertretern konkurrierender Fachverbände lähmend. Die Demutshaltung erschwerte die Aufrechterhaltung des Anspruchs auf Therapiekompetenz in allen Bereichen des Organfaches Haut. Beispielhaft kann in diesem Zusammenhang auf das Organfach Augenheilkunde verwiesen werden. Jeder Ophthalmologe würde es als Zumutung empfinden, die spezifischen Operationen seines Fachgebietes den Chirurgen überlassen zu müssen."

\section{Umblick}

\section{Kontinuität und Wandel in 25 Jahren}

Der Versuch, Kontinuität und Wandel der operativen und onkologischen Dermatologie in den verschiedenen Bereichen, ihre aktiven Behandlungsmaßnahmen bei Hauterkrankungen und ästhetischen Behinderungen darzustellen, muss sich auf wenige Beispiele beschränken. Die ganze „Spielbreite“ der Entwicklung ist in den bisher erschienenen 19 Bänden „Fortschritte der Operativen und Onkologischen Dermatologie" nachzulesen. Sie basieren auf den Referaten von 25 Jahrestagungen der Vereinigung für Operative und Onkologische Dermatologie, die in Deutschland, Österreich und der Schweiz stattfanden. Sie kennzeichnen die ganze Breite der wissenschaftlichen Tätigkeit, die schwerpunktmäßig die Ergebnisse manuellen Arbeitens, die operative Tätigkeit, herausstellt, ohne die konservative Therapie, wie sie z. B. in der Kontinuität nach erfolgter Operation beim malignen Melanom erforderlich ist, zu vernachlässigen. Die operative Therapie ist ein hervorragendes Beispiel dafür, dass fließende Über- gänge zu anderen Therapien und die Kombination verschiedener Therapien typisch sind.

Nicht ausbleiben konnte, dass sich verschiedene Bereiche der operativen Dermatologie, wie die onkologische Dermatologie, Phlebologie, Proktologie, ästhetisch-korrektive Therapie, Lasertherapie und Wundheilung um nur einige zu nennen, in eigenen Arbeitsgemeinschaften bis hin zu eigenen Gesellschaften formierten. Diese Entwicklung ist, weil interessengesteuert, nicht ungefährlich. Vor allem dann, wenn diese Bereiche dem lenkbaren Einfluss der Dermatologie entzogen werden und ein entsprechendes Eigenleben beginnen. Umso mehr hat die Vereinigung für Operative Dermatologie versucht, auf ihren Tagungen diese verschiedenen Bereiche zusammenzuführen. Das ist ihr, abhängig von der Thematik der Tagungen, z. T. hervorragend gelungen. Ohne Vollständigkeit zu erreichen, seien hier die folgenden Thematiken genannt:

- „Gegenwärtiger Stand der operativen Dermatologie“ (Wuppertal 1986),

- „Onkologische Dermatologie. Neue Aspekte. Altersbedingte Besonderheiten“(Würzburg 1991),

- „Wundheilung - Wundverschluss“ (Köln 1992),

- „Operative und konservative Dermato-Onkologie“ (Heidelberg 1994),

- „Operative und konservative Dermato-Onkologie im interdisziplinären Grenzbereich“ (Zürich 1995),

- "Operative onkologische Dermatologie“ (Kassel 1998),

- „Standards und Trends in der operativen und onkologischen Dermatologie, Phlebologie und Proktologie“(Dresden 2000).

Besondere Schwerpunkte wurden mit ausgewählten Erkrankungen und altersbezogenen Erkrankungen gesetzt, wie:

- „Das Basaliom“ (Zürich 1980 und Kassel 1992),

- „Komplikationen in der operativen Dermatologie“ (München 1983),

- „Diagnostik und Therapie maligner Melanome“ (Hamburg 1989),

- „Operative Dermatologie im Kindes- und Jugendalter (Regensburg 1996),

- „Ästhetische und korrektive Dermatologie“ (München 1997),

- „Krankheiten der Hautanhangsgebilde, Wund- und Narbenmanagement“" (Salzburg 1999).

Sehr früh waren die Tagungen mit praktischer Tätigkeit („Handson-Training“) in Operationskursen am Schweinebein und Schweinekopf sowie bei Demonstrationen ästhetisch-korrektiver Maßnahmen gekoppelt. Der Wunsch, operative Maßnahmen in Einzelschritten nachvollziehen zu können, wurde mit der Abfolge von OP-Einzelschritten im Diapositiv gut dokumentiert, reichte aber gerade den jüngeren operativ Tätigen nicht aus. So entstand neben, dann schließlich als eigenständige Veranstaltung das OP-Live-Symposium in Darmstadt. Den Initiatoren um M. Hagedorn sind hervorragende Veranstaltungen zur operativen Dermatologie des Dermatologen im Sinne von Kleine-Natrop und Tritsch bis Landes zu verdanken. Die (Über-)Betonung kosmetischer Maßnahmen und die Internationalität darf als Tribut an die Wünsche „unserer Kunden“ verstanden werden. Noch ist die operative Dermatologie definiert als Teilbereich der Dermatologie zur Behandlung definierter Krankheiten. Dazu gehört z. B. die Behandlung der benignen symmetrischen Lipomatose mit- 
tels Liposuktion, einer Technik, die der operative Dermatologe erlernen und dafür einsetzen sollte [20]. An dieser Stelle ist die Unterscheidung der Begriffe Ästhetik und Kosmetik im Zusammenhang mit der operativen Tätigkeit notwendig. Ästhetik ist das Streben nach einem Normalzustand, der sich an einem idealen Schönheitsbild, wie z. B. in der griechischen Antike, misst. Damit ist der Begriff mehr oder weniger zeitlos. So sollten der Ästhetischen Chirurgie die Korrektur von Normabweichungen zugeordnet werden, die durch angeborene Fehlbildungen oder durch krankheitsbedingte bzw. traumatische Fehlentwicklungen entstanden sind. Im Gegensatz dazu ist der Begriff Kosmetik der herrschenden Mode unterworfen und wandelbar. Die Kosmetische Chirurgie kommt daher dem Wunsch des „Patienten“ („Kunden“) entgegen, seine subjektiven Vorstellungen vom eigenen Erscheinungsbild zu verwirklichen und sich durch chirurgische Korrektur dem augenblicklich herrschenden Zeitgeschmack anzugleichen. Lassen wir den Bereich der Kosmetik zu der tragenden Säule der Dermatologie werden, vergrößert sich die Gefahr, unser Fach im Konkurrenzkampf der Fächer zu verlieren. Deshalb ist zu begrüßen, dass die nächste VOD-Tagung Ende Oktober 2004 gemeinsam mit dem OP-Live-Symposium stattfindet. Videosequenzen operativer Verfahren werden Vorträge ergänzen, Live-Operationsdemonstrationen zur Behandlung von Hauterkrankungen gemeinsam mit der kosmetischen Chirurgie dargestellt werden. Ziel muss es sein, den Weiter- und Fortbildungscharakter der operativen Therapie des Dermatologen ausgewogen und umfassend zu garantieren.

\section{Entwicklungslinien der operativen Therapie über drei Jahrzehnte}

Im Folgenden dürfen ausschnittweise die Kontinuität und der Wandel in verschiedenen Arbeitsrichtungen der operativen Therapie über die Jahre dargestellt werden, an denen besonders Dermatologen Anteil hatten. Inhaltlich basieren sie auf den gehaltenen Referaten der Jahrestagungen der VOD, wobei bewusst auf umfangreiche Literaturhinweise verzichtet wird, die der Interessierte in den 19 VOD-Bänden findet.

\section{Plastisch-rekonstruktive und andere aktive Verfahren}

Die meisten auch heute verwendeten Verfahren zur Rekonstruktion operativ gesetzter Defekte nach Tumorentfernung bewährten sich bereits Anfang der 70er-Jahre des vorigen Jahrhunderts. Neuere Lappenplastiken, wie die Treppenplastik zur Rekonstruktion von ausgedehnten Unterlippendefekten, subkutan gestielte (Gleit-)Lappen und fasziokutane Lappen wurden in den 80erJahren rasch in das operative Repertoire aufgenommen [14,22], Indikationen und Therapieerfolge mit dem umgedrehten Koriumtransplantat ab 1986 dokumentiert. Ab 1990 werden für ausgewählte Indikationen Hautexpander zur Hautdehnung eingesetzt. Bewährt haben sie sich in den folgenden Jahren besonders am Kopf und im Stammbereich. Die Entwicklung muskulokutaner Lappen und freier mikrovaskulärer Transplantate in den 80er-Jahren verfolgte die operative Dermatologie mit und akzeptiert hier ihre eigenen Grenzen. Die Weiter- und Neuentwicklung chirurgischer Instrumente und Nahtmaterialien machte neben der instrumentellen Knüpftechnik neue Nahttechniken, wie fortlaufende vollständig versenkte Nähte (1986) und die als „Schmetterlingsnähte“ bekannt gewordene, versenkte subkutankoriale Naht als Voraussetzung für eine „cicatrix optima“ (1990) möglich. Der Wandel in der Therapie der axillären Hyperhidro- sis, der konnatalen Riesenpigmentnävi und der Nagelfehlbildungen ist beispielhaft für die operative Therapie des Dermatologen. Wurde die axilläre Hyperhidrosis 1975 noch großzügig exzidiert, folgte bald die schmale Exzision in Kombination mit der noch offenen Kürettage, um schließlich von der rein subkutanen Kürettage, ausgehend von einem kleinen Hautschnitt und mit Liposuktionskanülen in Tumeszenzanästhesie, abgelöst zu werden. Heute haben sowohl die Botulinumtoxin-Injektion als auch die Kürettage ihre Indikationen. Mit der Behandlung der konnatalen Riesenpigmentnävi verbindet sich die Erfolgsstory der flächenhaften Dermabrasion, die in den 70er-Jahren als Frühbehandlung in den ersten Lebensmonaten zur therapeutisch befriedigenden Aufhellung propagiert, 1996 mit großen Fallzahlen unterlegt wurde [17]. In den letzten Jahren wird versucht, eine weitere Aufhellung der verbliebenen Pigmentierung mit dem Er:YAG-Laser zu erreichen. Mit der lateralen, segmentalen Nagelmatrixexzision zur Behandlung eingewachsener Nägel verbindet sich seit 1978 der Name E. Haneke [5]. Seine Methode, 1985 um die Phenolätzung als Kaustik des lateralen Nagelhorns erweitert, ist sie heute von den Chirurgen anerkannt. 1982 berichteten Arbeitsgruppen aus Bochum, Dresden, Hamburg und Hornheide erste größere Fallzahlen zur erfolgreichen Behandlung von angiomatösen Neubildungen, gutartigen Tumoren und Basalzellkarzinomen mittels Kryotherapie in der Technik des contact- und sprayfreezings. Heute hat die Kryotherapie mit flüssigem Stickstoff neben der Therapie mit dem Farbstofflaser zur Frühtherapie kindlicher Hämangiome ihre Indikation, ebenso im Rahmen der Polypragmasie bei hypertrophen Narben und Keloiden sowie zur Behandlung von Basalzellkarzinomen und kutanen Melanommetastasen.

Untrennbar mit der operativen Dermatologie verbunden ist die Entwicklung der mikrographischen Chirurgie (Synonym: mikrographisch-kontrollierte Chirurgie, histographische Chirurgie), die G. Burg Anfang der 70er-Jahre aus den USA nach München mitbrachte und mit dem Operateur B. Konz für den Einsatz in der Chirurgie des Basalzellkarzinoms perfektionierte [4]. 1982 stellt $H$. Breuninger die routinemäßige histologische Randschnittkontrolle mit vertikal angefertigten Schnitten vor [3]. Später folgen die verschiedensten Variationen von der „Tübinger Torte“ über die „einfache Flunder“ bis zur „doppelten Flunder“. Die histographische Chirurgie, die zwischenzeitlich Standard in der Mund-Kiefer-Gesichtschirurgie sowie der Hals-Nasen-Ohrenheilkunde ist, darf als ein typisches Beispiel für Ausstrahlung auf andere operative Fachgebiete gelten. Möglich wurde sie nur durch die gelungene Verknüpfung von dermatohistopathologischer und operativer Sichtweise.

Nicht weniger bedeutsam für die operative Dermatologie und propagiert von operativen Dermatologen wurden in den vergangenen 10 Jahren neben der klassischen Infiltrationsanästhesie mit der Injektion kommerziell hergestellter Anästhetika über eine Spritze, die als Nachteil das begrenzte injizierbare Volumen und damit die begrenzte anästhesierbare Fläche aufweisen, die Tumeszenzanästhesie als Tumeszenz-Lokalanästhesie (TLA) von J. Klein zuerst in den USA für die Liposuktion, von G. Sattler und B. Sommer sowie als subkutane Infusionsanästhesie (SIA) von $\mathrm{H}$. Breuninger für den deutschsprachigen Raum mit verschiedenen, mengenmäßig steuerbaren Pumpensystemen wiederbelebt und weiterentwickelt. Heute finden die TLA und die SIA in der gesam- 
ten Breite der operativen Dermatologie Anwendung. Beide Verfahren gestatten, gepufferte handelsübliche Anästhetika oder hochverdünnte Mischanästhetikalösungen druck- und volumenkontrolliert über große Flächen mit speziell entwickelten Pumpensystemen oder handelsüblichen bzw. modifizierten Infusomaten subkutan schmerzarm zu verteilen $[19,23]$.

Die operative Proktologie, einst ein therapeutischer Schwerpunkt in der Dermatologie, wird bis heute kontinuierlich in einzelnen Hautkliniken (z. B. Aachen, Darmstadt, Heidelberg) und ambulant in verschiedenen spezialisierten Niederlassungen durchgeführt. Während die kleinen Eingriffe zur Behandlung der perianalen Thrombose und akuten Analfissuren, des periproktitischen Abszesses und der Marisken sowie spitzer Kondylome zu den täglichen Routinebehandlungen gehören, sind größere und große Eingriffe bei chronischer Analfissur, Hämorrhoiden 3. Grades, Perianalzonenmalignomen, dem anorektalen Abszess, der Analfistel und dem Analkanalmalignom mehr oder weniger Arbeitsgebiete chirurgischer Proktologen. Modernere Verfahren, z. B. zur Hämorrhoidektomie mit dem Zirkular-Stapler, werden bis auf wenige Ausnahmen nicht (mehr) an der dermatologischen Einrichtung gelehrt und z. B. Instituten für Enddarmerkrankungen überlassen.

Als 1985 ausführlich die Vorteile der Wundkonditionierung und der sekundären Wundheilung in Kassel dargestellt werden, wird auf eine alte Tradition in der Chirurgie zurückgegriffen aber gleichzeitig das Thema „Chronische Wunden, Wundheilung, Wundauflagen" auf den folgenden Tagungen auf den Weg gebracht. 1992 ist auf der Kölner Tagung (G. Mahrle) die Wundheilung mit ihren zellulären und molekularen Grundlagen der Schwerpunkt (Arbeitsgruppe um T. Krieg) [14]. Tissue-Engineering, immer wieder neue Wundauflagen und in neuerer Zeit Wundmanager haben ihre Grenzen dort, wo vor der definitiven operativen Versorgung chronischer Problemwunden Vakuumversiegelungstechniken vorgeschaltet werden. Die Behandlung der chronischen Wunden, und dazu gehört expressis verbis das chronische Beingeschwür, wird schwerpunktmäßig in der Dermatologie bleiben, wenn wir chronische Wunden nicht nur mit Wundauflagen konditionieren sondern definitiv, betont operativ versorgen können. Dazu sollten die Podien der Tagungen der DDG, der VOD, der Deutschen Gesellschaft für Phlebologie und der Deutschen Gesellschaft für Wundheilung und Wundbehandlung genutzt werden.

1982 tragen Urologen auf der Münchener Tagung ihre Ergebnisse zur Photo-Chemo-Therapie mit Hämatoporphyrin-Derivat (HpD) bei Blasentumoren vor. 10 Jahre später in Kassel und ab 1995 ist die photodynamische Diagnostik und - Therapie eine regelmäßige Thematik einer heute zugelassenen Therapie bei Präkanzerosen und (superfiziellen) Basalzellkarzinomen.

\section{Problemtumor malignes Melanom}

1975 gelten in der Therapie des damals seltenen malignen Melanoms seine radikale Exzision, nach Möglichkeit mit $5 \mathrm{~cm}$ seitlichem Sicherheitsabstand, und die postoperative Radionuklidtherapie der Extremitätentumoren als Standard. Als 1982 ein Sicherheitsabstand von maximal $3 \mathrm{~cm}$ diskutiert wird, ist das Resümee „... inwieweit sich dieser Standpunkt durchsetzen wird, bleibt abzuwarten“ [16]. 1984 werden die Ergebnisse einer adjuvanten
Therapie mit DTIC und BCG vorgetragen [1]. Die damals noch nicht vorhandenen modernen Antiemetika führen dazu, dass $68 \%$ aller DTIC-Behandlungen vorzeitig abgebrochen werden. Operiert wird weiter mit großem Sicherheitsabstand, gefolgt von elektiver Lymphknotendissektion und Kontinuitätsdissektion. Zukunftsweisende Referate heben die Bedeutung statistischer Methoden und die Erarbeitung von Studienprotokollen für onkologische Therapien ebenso hervor wie eine verbesserte Melanomklassifikation und -nachsorge. 1986 ist die 1981 begonnene Epilumiszenzmikroskopie (Auflichtmikroskopie) praxisreif, die Lymphabstromszintigraphie bildet die Leitschiene der Kontinuitätsdissektion [27]. Nach Hamburg 1989 mit dem Generalthema „Diagnostik und Therapie maligner Melanome“, das eine ganze Tagung ausfüllt, machen Würzburg 1991, Heidelberg 1994 und Zürich 1995 deutlich, dass die Erkenntnisse molekularer, biologischer und pathophysiologischer Grundlagen, die erweiterte Diagnostik mittels Sonographie und Positronenemissionstomographie, statistische Fragestellungen und Chemo-Immuntherapie-Studienprotokolle eine Arbeitsgemeinschaft Onkologische Dermatologie rechtfertigen [24]. Der operative Dermatologe hat die radikale Therapie des primären malignen Melanoms zugunsten einer der Tumordicke angepassten Exzision verlassen und um die Wächter-Lymphknoten-Dissektion [8] als diagnostische Maßnahme erweitert. Ihrem Charakter entsprechend wird die VOD auch in Zukunft ihren onkologischen Melanompart pflegen und eng mit der Arbeitsgemeinschaft Dermatologische Onkologie (ADO) zusammenarbeiten.

\section{Operative Phlebologie}

Unabhängig von der Gründung der Deutschen Gesellschaft für Phlebologie, die berechtigterweise von Dermatologen mitgetragen wird, sind operative Dermatologen über mehr als 25 Jahre Sachverwalter der Kontinuität und Protagonisten des Fortschritts sowohl in der Diagnostik als auch operativen Therapie. Sie haben selbst operative Verfahren entwickelt und neue Erkenntnisse rasch realisiert. Die frühen Jahre des klassischen operativen Vorgehens bei chronischer Veneninsuffizienz (CVI) verbinden sich mit dem Namen K. Salfeld. Als W. Hach 1981 die selektive Saphenaresektion einführt, folgen seinem Konzept rasch die operativen Dermatologen. Die Fortschritte der Venendiagnostik (z. B. Licht-Reflexions-Rheographie oder Duplexsonographie) gehen parallel mit neuen Operationstechniken der frühen 90er-Jahre, wie invaginierendes Stripping, Pin-Stripping oder ein Kryo-Stripping (zur Vermeidung eines zweiten Hautschnittes). Die Therapie insuffizienter Perforanten wird um die endoskopische subfasziale Perforansdiszision erweitert, die Ulkustherapie durch die paratibiale Fasziotomie, später durch die (radikale) Fasziektomie verbessert. K. H. Galli und H. Wolf (Nürnberg) berichten 1990 erstmals über die guten Ergebnisse nach Abtragung des liposklerotischen Bindegewebes im Ulkusbereich als so genanntes Shaving, W. Schmeller stellt das Verfahren auf eine wissenschaftlich begründete Basis. Die besondere ästhetische Verantwortung des Dermatologen für die Haut hat in der operativen Phlebologie dazu geführt, dass die „KleinschnittTechnik" ebenso Standard geworden ist, wie das Arbeiten in Blutleere und seit 1994 der Übergang von der Allgemein- und Regionalanästhesie zur heute weit verbreiteten Tumeszenzanästhesie. Die farbkodierte Duplexsonographie (FKDS), Goldstandard für das Detektieren insuffizienter Perforantes hat die modernen Diagnostikverfahren komplettiert. Innovative Operati- 
onsverfahren, wie die endovenöse Obliteration der Stammvarikose mit Radiowellen (VNUS-Closure), die Venenobliteration mit dem 810 und $940 \mathrm{~nm}$ Diodenlaser und die Mikroschaumverödung werden sich im therapeutischen Reigen positionieren.

Idealerweise liegen Diagnostik, operative Planung und Ausführung in einer Hand und zwar der des konservativ und operativ ausgebildeten operativen Dermatologen/Phlebologen.

\section{Lasertherapie}

Nachdem die Arbeitsgruppe um M. Landthaler 1981 im „Hautarzt" [12] kritisch zu den Indikationen des Lasers Stellung nimmt, haben die Protagonisten des Lasereinsatzes in der operativen Dermatologie 1982 erstmalig und dann auf jeder Jahrestagung der VOD Gelegenheit, Grundlagen und Indikationen des sich von Jahr zu Jahr ausweitenden Laserparkes vorzutragen (z. B. M. Drosner, U. Hohenleutner, R. Kaufmann, M. Landthaler, M. Podda und C. Raulin) [6,7]. Heute haben die Farbstofflaser ihre klare Indikation, z. B. bei den angeborenen Gefäßfehl- und -neubildungen. Gepulste $\mathrm{CO}_{2}$-Laser und der Er:YAG-Laser werden zur Behandlung von Falten und Fältchen eingesetzt. Die anfängliche Euphorie eines angeblich nebenwirkungsfreien Laserskin-Resurfacing um 1995 ist abgeklungen und sachlicher Argumente gewichen [26]. Zur Zeit stehen die Indikationen für den Einsatz der Blitzlampen auf dem Prüfstand.

\section{5. Ästhetische und korrektive Dermatologie}

1984 stellt B. Konz auf der Lübecker VOD-Tagung erstmals ein injizierbares Kollagen zur Hautaugmentation bei krankhaften Veränderungen und zur Korrektur kosmetisch störender Falten vor [9]. 13 Jahre später wird in München die 20. Jahrestagung der ästhetischen und korrektiven Dermatologie gewidmet sein. Zwischenzeitlich sind ästhetische Eingriffe und korrektive Maßnahmen an der Haut zu einem „heiß umkämpften, merkantilen Markt" geworden. Erneut wird aus der Ecke der plastischen Chirurgen gegen die operative Tätigkeit des Dermatologen polemisiert, Kosmetikinstitute versuchen, einen Teil des lukrativen Kuchens abzubekommen. Für den operierenden Dermatologen sind bei der Behandlung maligner Tumoren und benigner Hautveränderungen ästhetisch-korrektive Aspekte von Bedeutung, doch muss der kurative Erfolg meist das Primat besitzen. Durch die Einführung neuerer Verfahren, wie die Anwendung injizierbarer und implantierbarer Eigen- und Fremdmaterialien (Hautaugmentation), der Hauterneuerung durch Chemical-Peeling und Laserskin-Resurfacing sowie der Liposuktion in Tumeszenzlokalanästhesie (an deren Entwicklung zur Praxisreife deutschsprachige Dermatologen, betont operativ tätige Fachärzte beteiligt waren) sah sich die VOD berechtigt, diese Methoden darzustellen, kritisch zu würdigen und auch bis heute verantwortungsvoll mitzutragen [20]. Unser Fachgebiet muss die ästhetisch-korrektiven Verfahren, die von ihm mit entwickelt wurden (1956 gründete H. Th. Schreus die „Deutsche Gesellschaft für ästhetische Medizin und ihre Grenzgebiete“), im Rahmen ihres Weiter- und Fortbildungsauftrages weitergeben. Es zeigt sich immer wieder, dass plastische Chirurgen wohl eine Nase richten können aber von den Feinheiten der Behandlung und Korrektur der Gesichtshaut wenig verstehen, eben weil sie keine Dermatologen sind. Diesen Vorteil sollte unser Fach nutzen. Gut weitergebildet wird der Hautfacharzt den Ratsuchenden umfassend beraten, behandeln und im Bedarfsfall bei entsprechendem Wissen, das auf
Fortbildungen angeboten wird, eine für den Patienten optimale Behandlungseinrichtung empfehlen [10]. G. Krieger, seit Beginn unserer Tagungen als juristischer Berater tätig, hat es 1997 auf den Punkt gebracht und führt u. a. aus ,... wie bereits ausgeführt, schuldet der operierende Arzt keinen bestimmten Operationserfolg, denn er kann für den Erfolg seiner Behandlung nicht garantieren. Er schuldet jedoch eine fachgerechte Behandlung. Die Wahl der Behandlungsmethode ist im Rahmen der Therapiefreiheit Aufgabe des Arztes. Ihm obliegt die Verantwortung und Beurteilung, wie er der Krankheit oder dem ästhetischen Verlangen des Patienten gerecht werden will. Der Arzt wird nicht nur nach seinem eigenen Können und seinem individuellen ärztlichen Wissen beurteilt, sondern von ihm wird ein bestimmter Mindeststandard erwartet. In der Rechtssprechung ist dies der Standard eines erfahrenen Facharztes. Dabei wird im Rahmen der Qualitätssicherung von ihm auch entsprechende Fortbildung verlangt.“ [11]

\section{Ausblick}

Nach 30 Jahren ist das Fach Haut- und Geschlechtskrankheiten in Deutschland mit einer starken operativen Dermatologie ausgerüstet, die alle Facetten aktiven Handelns abdeckt. Wie in jedem Fach, haben sich für verschiedene Bereiche über das Land verstreut Schwerpunkte entwickelt, die neben der Pflicht eine hervorragende operative Kür bieten. Was ist also in Zukunft zu tun, um die operative Tätigkeit auf einem hohen Standard zu halten und weiterzuentwickeln?

Neben der Präsentation der Ergebnisse operativer Arbeit auf wissenschaftlichen Tagungen der VOD und der DDG sowie auf internationaler Ebene, der Weiter- und Neuentwicklung von Leitlinien, der engeren Zusammenarbeit mit der Deutschen Dermatologischen Gesellschaft und dem Berufsverband Deutscher Dermatologen, dem Bekanntmachen operativer Arbeit in den Medien, liegt der Schwerpunkt der nächsten Jahre darin, eine flächendeckende, hohe Qualität der operativen und onkologischen Tätigkeit des Dermatologen in Praxis und Klinik zu sichern. Dafür ist ein Hospitationsprogramm von der VOD gemeinsam mit der DDG erarbeitet worden. Bisher sind von 40 Kliniken positive, die Initiative unterstützende, Rückmeldungen eingegangen. Operativ ausgewiesene dermatologische Praxen sind zur Mitarbeit bereit, so dass im Dezember 2003 auf einer Sitzung des erweiterten Vorstandes der VOD eine erste Fassung eines Hospitationsprogramms als Grundlage für die Startphase des RotationsHospitationsprogramms verabschiedet wurde. Dieses wird in den nächsten Monaten veröffentlicht, sodass an dieser Stelle abschließend die wichtigsten Ziele wiedergegeben werden:

- Qualitätssicherung durch praxisnahe Vermittlung von Wissen und OP-Techniken bei dermatologisch relevanten Erkrankungen, einschließlich Fehl- und Neubildungen,

- Zertifizierung operativer Bereiche in Klinik und Niederlassung durch die VOD und DDG,

- Erhöhung von Qualität und Quantität in der operativen Ausbildung,

- Prozessoptimierung operativer Bereiche - auch im Hinblick auf DRG-basierte Abrechnungssysteme,

- Engere kollegiale Zusammenarbeit und Austausch zwischen den Dermatologischen Kliniken und den niedergelassenen Dermatologen mit dem Schwerpunkt operative Dermatologie, 
- Kooperation mit der Arbeitsgemeinschaft Dermatologische Onkologie der DDG auf dem Gebiet der konservativen Therapie von Hauttumoren (Leitlinienentwicklung, Austausch der Studienprotokolle, gemeinsame Strategieplanung der onkologischen Dermatologie),

- Definition von Qualitätskriterien, OP-Standards in der operativen Dermatologie,

- Zertifizierte Ausbildungskurse bei den Tagungen der DDG, des BVDD sowie im Rahmen von Tagungen und Strategiesitzungen der VOD,

- Erhöhung der Akzeptanz der operativen Dermatologie durch andere operative Fachgebiete.

Die Weiterbildung zum Arzt für Haut- und Geschlechtskrankheiten muss eine definierte Ausbildung in operativer und onkologischer Dermatologie beinhalten. Was heute in einem Fach nicht mehr gelehrt und angeboten wird, dies ist morgen dem Fach verloren und findet sich dann sehr rasch in anderen Fächern wieder!

\section{Literatur}

${ }^{1}$ Braun-Falco O, Landthaler M, Hohenleutner U, Hohenleutner S, Hölzel D, Konz B, Schmoeckel Ch. Adjuvante Therapie maligner Melanome im Stadium I. In: Wolff HH, Schmeller W (eds). Fehlbildungen, Nävi, Melanome. Fortschritte der operativen Dermatologie, Bd. 2. Berlin Heidelberg, New York: Springer, 1985: 268-273

${ }^{2}$ Braun-Falco O. Heutiger Stellenwert der Operativen Dermatologie (Geleitwort). In: Wolff AA, Schmeller W (eds). Fehlbildungen, Nävi, Melanome. Fortschritte der operativen Dermatologie, Bd. 2. Berlin, Heidelberg New York: Springer, 1985: V - VIII

${ }^{3}$ Breuninger $\mathrm{H}$. Histologic control of excised tissue edges in the operative treatment of basal-cell carcinomas. J Dermatol Surg Oncol 1984; 10: $724-727$

${ }^{4}$ Burg G. Mikroskopisch kontrollierte (histographische) Chirurgie. In: Konz B, Burg G (eds). Dermatochirurgie in Klinik und Praxis. Berlin, Heidelberg, New York: Springer, 1977: 72 - 82

${ }^{5}$ Haneke E. Chirurgische Behandlung des Unguis incarnatus. In: Salfeld (ed). Operative Dermatologie. Berlin, Heidelberg, New York: Springer, 1979: $185-188$

${ }^{6}$ Hohenleutner U, Landthaler M. Welche Laser gibt es für die Hauterneuerung? In: Konz B, Wörle B, Sander CA (eds). Ästhetische und korrektive Dermatologie. Fortschritte der operativen und onkologischen Dermatologie, Bd. 14. Berlin, Wien: Blackwell, 1999: 265-270

${ }^{7}$ Kaufmann R. Erfahrungen mit dem Erbium:YAG-Laser. In: Konz B, Wörle B, Sander CA (eds). Ästhetische und korrektive Dermatologie. Fortschritte der operativen und onkologischen Dermatologie, Bd. 14. Berlin, Wien: Blackwell, 1999: 271 - 278

${ }^{8}$ Koller J, Gmeiner D, Kiessler J, Rattenbacher L. Sentinel-LymphknotenDissektion. In: Rompel R, Petres J (eds). Operative onkologische Dermatologie. Fortschritte der operativen und onkologischen Dermatologie, Bd. 15. Berlin, Heidelberg, New York: Springer, 1999: 226 - 230

9 Konz B. Injizierbares Kollagen: Indikationen und Kontraindikationen. In: Wolff HH, Schmeller W (eds). Fehlbildungen, Nävi, Melanome. Fortschritte der operativen Dermatologie, Bd. 2. Berlin, Heidelberg, New York: Springer, 1985: 159-166

${ }^{10}$ Konz B. Operative Dermatologie im Grenzbereich. In: Tilgen W, Petzoldt D (eds). Operative und konservative Dermato-Onkologie. Neue Ansätze und Strategien. Fortschritte der operativen und onkologischen Dermatologie, Bd. 10. Berlin, Heidelberg, New York: Springer, 1995: $323-327$

${ }^{11}$ Krieger G. Juristische Aspekte bei ästhetischen Operationen: In: Konz B, Wörle B, Sander CA (eds). Ästhetische und korrektive Dermatologie.
Fortschritte der operativen und onkologischen Dermatologie, Bd. 14. Berlin, Wien: Blackwell, 1999: 323-329

${ }^{12}$ Landthaler M, Haina D, Waidelich W, Braun-Falco O. Behandlungen mit einem Argonlaser in der Dermatologie. Hautarzt (Suppl) 1981; 5: 433-435

${ }^{13}$ Macher E. Das maligne Melanom - Fortschritt durch Forschung. In: Tilgen W, Petzoldt D (eds). Operative und konservative Dermato-Onkologie. Neue Ansätze und Strategien. Fortschritte der operativen und onkologischen Dermatologie, Bd. 10. Berlin, Heidelberg, New York: Springer, 1995: 1 -9

${ }^{14}$ Mahrle G, Schulze H-J, Krieg T (eds). Wundheilung-Wundverschluss. Theorie und Praxis chirurgischer und konservativer Maßnahmen. Fortschritte der operativen und onkologischen Dermatologie, Bd. 8. Berlin, Heidelberg, New York: Springer, 1994

${ }^{15}$ Müller RPA, Konz B. Zur Lage der operativen und onkologischen Dermatologie. In: Konz B, Müller RPA (eds). Zur Lage der operativen und onkologischen Dermatologie. Fortschritte der operativen und onkologischen Dermatologie, Bd. 13. Berlin, Wien: Blackwell, 1997: 1 - 5

${ }^{16}$ Petres J, Müller RPA. Strategie der operativen Therapie des malignen Melanoms. In: Petres J (ed). Aktuelle Behandlungsverfahren. Fortschritte der operativen Dermatologie, Bd. 3. Berlin, Heidelberg, New York: Springer, 1987: 119-133

${ }^{17}$ Petres J, Rompel R. Konnatale Nävuszellnävi. In: Burg G, Hartmann AA, Konz B (eds). Onkologische Dermatologie. Neue Aspekte. Altersbedingte Besonderheiten. Fortschritte der operativen und onkologischen Dermatologie, Bd. 7. Berlin, Heidelberg, New York: Springer, 1992: $220-229$

${ }^{18}$ Salfeld K. Welches Ausmaß operativer Tätigkeit ist in einer Hautklinik vertretbar? In: Konz B, Burg G (eds). Dermatochirurgie in Klinik und Praxis. Berlin, Heidelberg, New York: Springer, 1977: 52 - 56

${ }^{19}$ Sattler G, Rapprich S, Hagedorn M. Tumeszenzlokalanästhesie Untersuchungen zur Pharmakokinetik von Prilocain. H + G 1998; 7: $522-525$

${ }^{20}$ Sattler G, Sommer B. Liposuktion und Lipaugmentation: Was gibt es Neues? In: Augustin M, Peschen, M, Peters J, Schöpf E. (eds). Innovation und Qualität in der operativen Dermatologie. Fortschritte der operativen und onkologischen Dermatologie, Bd. 18. Berlin: congress compact, 2003: 85-92

${ }^{21}$ Scholz A. Geschichte der operativen Dermatologie in Deutschland. In: Zur Lage der operativen und onkologischen Dermatologie. Fortschritte der operativen und onkologischen Dermatologie, Bd. 13. Berlin, Wien: Blackwell, 1997: 9-40

${ }^{22}$ Sebastian G. Funktionelle und ästhetische Spätergebnisse nach operativer Behandlung von Unterlippentumoren. In: Haneke E (ed). Gegenwärtiger Stand der operativen Dermatologie. Fortschritte der operativen Dermatologie, Bd. 4. Berlin, Heidelberg, New York: Springer, 1988: $108-121$

${ }^{23}$ Sommer B, Sattler G, Hanke CW. Die Tumeszenzlokalanästhesie. Berlin, Heidelberg, New York: Springer, 1991

24 Tilgen W, Strauss LG, Welters H, Brado B, Zierott U, Helus F, Mende U, Petzoldt D. Neueste Konzepte in der Diagnostik und Therapie des malignen Melanoms. Hautarzt 1990; 41 (Suppl X): 133-137

25 Tritsch H. Aus den frühen Jahren der VOD. In: Konz B, Müller RPA (eds). Zur Lage der operativen und onkologischen Dermatologie. Fortschritte der operativen und onkologischen Dermatologie, Bd. 13. Berlin, Wien: Blackwell, 1997: 157-162

${ }^{26}$ Wiest L. Wann sind welche Behandlungsmethoden für welche Falten sinnvoll? In: Sebastian G, Stein A, Hackert I (eds). Standards und Trends in der operativen und onkologischen Dermatologie, Phlebologie und Proktologie. Fortschritte der operativen und onkologischen Dermatologie Bd. 17. Berlin: congress compact, 2001: 17-24

${ }^{27}$ Winter H, Bellmann K-P, Audring H, Küchler I, Garbe C. Prognoseverbesserung durch Kontinuitätsdissektion nach Lymphabstromszintigraphie bei Rumpfmelanomen. In: Winter H, Bellmann K-P (eds). Operative Dermatologie. Fortschritte der operativen und onkologischen Dermatologie, Bd. 9. Berlin, Heidelberg, New York: Springer, 1995: $251-258$ 